\title{
Breast tumor segmentation in mammography image via Chan-Vese technique
}

\author{
Eman A. Radhi, Mohammed Y. Kamil
}

College of Sciences, Mustansiriyah University, Baghdad, Iraq

\begin{tabular}{l}
\hline Article Info \\
\hline Article history: \\
Received Jan 23, 2021 \\
Revised Mar 13, 2021 \\
Accepted Mar 21, 2021 \\
\hline
\end{tabular}

\section{Keywords:}

Active contour

Breast tumour

Chan-Vese

Mammography

Segmentation

\begin{abstract}
The accurate segmentation of tumours is a crucial stage of diagnosis and treatment, reducing the damage that breast cancer causes, which is the most common type of cancer among women, especially after the age of forty. The task of segmenting breast tumours in mammograms is very difficult, as its difficulty lies in the lack of contrast between the tumour and the surrounding breast tissue, especially when dealing with small tumours that are not clear boundaries and hidden under the tissues. As algorithms often lose an automatic path toward the boundaries of the tumour at try to determine the site of this type of tumour. The study aims to create a clear contrast between the tumour and the healthy breast area. For this purpose, we used a Gaussian filter as a preprocessing as it works to intensify the low-frequency components while reducing the high-frequency components as the breast structure is enhanced and noise suppression. Then, CLAHE was used to improve the contrast of the image, which increases the contrast between the tumour and the surrounding tissue and sharpens the edges of the tumour. Next, the tumour was segmented by using the Chan-Vese method with appropriate parameters defined. The proposed method was applied to all abnormal mammogram images taken from a publicly available mini-MIAS database. The proposed model was tested in two ways, the first is statistical that got results $(90.1,94.8,95.5,92.1,99.5)$ for Jaccard, Dice, PF-Score, precision, and sensitivity respectively. And the other is based on the segmented region's characteristics that results showed the algorithm could identify the tumour with high efficiency.
\end{abstract}

This is an open access article under the CC BY-SA license.

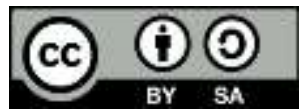

\section{Corresponding Author:}

Mohammed Y. Kamil

College of Sciences,

Mustansiriyah University, Baghdad, Iraq

Email: m80y98@uomustansiriyah.edu.iq

\section{INTRODUCTION}

Breast cancer continues to rise in developed countries, just as it is in developing countries. It is one of the most common types of cancer affecting women. Eighty percent is the rate of breast cancer survivors in countries with high incomes, while 40 percent in countries with low incomes [1]. "Magnetic resonance imaging" (MRI) [2], "computed tomography"[3], "mammography" [4] "ultrasound". These are medical imaging methods used for early screening and detection of breast cancer [5]. Mammography is an imaging of the breast using low energies of X-rays of about $30 \mathrm{kV}$ which produce hi-res images that can encode more shades provide the ability to detect abnormalities hidden within the breast and surrounding tissue overlapping [6]. Despite the limitations, a mammogram will diagnose $85 \%$ to $90 \%$ of breast cancer cases at least two years ago [7]. Segmentation of medical images is the method of defining the portion of the image that is not normal and interesting and maybe anomalies (for example, tumours) or part of the body parts [8]. One of the most significant stages of image processing is image segmentation. as it results in the rest of the processors' 
efficiency as it divides the image into separate regions depending on the amount of similarity and difference in features (for example texture, intensity, colour) [9]. Medical experts have a model of the object to be divided in their minds so they can define. Model-based segmentation methods attempt to simulate a specialist's knowledge and translate it into intelligent algorithms that have prior knowledge of and appearance of the object of interest. Therefore, model-based methods have higher segmentation accuracy than others [10]. Among the model-based methods, the deformation-based model method "active contour model" (ACM) is considered one of the powerful methods of computer-assisted medical image segmentation. The reason for this method's strength is its high potential to segment and trace the boundaries of the object of interest, as it can regulate the shape of the contour through the restrictions it imposes on it where it uses constraints (top to bottom) inferred from image information and (bottom to top) for the location, shape and size of this object [11]. The active contour can be divided into two basic approaches: (1) snakes [12] which are represented as explicit parameterized segmentation curves (e.g., parametric). (2) geometric active contours [13] which are represented as level sets based on the implicit representation of curves.

A literature review has found that it does not do well for images with intensity inhomogeneity, despite having a wider convergence spectrum and effectively handling topological changes using the CVM. The key explanation is that the CVM is built on the premise that image intensities have stayed constant in each region, leading to the incorrect movement of the contour [14]. S. N. Acho, et al. selected 50 mammograms obtained from a Hologic Selenia ${ }^{\mathrm{TM}}$ Dimensions $^{\mathrm{TM}}$ system to test their proposal in which they used the particle swarm optimization method in which an optimized mass-specific threshold value obtained from the probability matrix of the mass is calculated for the active convex contour. The mean similarity index for Jaccard was $0.89 \pm 0.071$ [15]. M. Mustafa, et al. applied the CVM to 30 mammograms from the DDSM Dataset after pre-processing the image with a Gaussian filter and then improving the contrast of the images. The result shows a $90 \%$ accuracy [16]. S. Soomro, et al. proposed a SPF function is characterized by a phaseshifted Heaviside function, which helps to achieve the optimal solution in fewer iterations. The suggested approach is tested on many mini-MIAS Dataset mammogram images. Sensitivity indices were 0.9865 [17]. M. Hmida, et al. suggested a fuzzy-energy-based model that is used for final mass delineation Where was the development of fuzzy contours and calculation of fuzzy membership maps of various groups in the image from the mini-MIAS dataset; incorporation of these maps into the CVM. The experimental findings indicate that with a precision of 88.08 per cent, the process reaches an overall true positive score of 91.12 per cent [18]. S. Soomro et al. proposed a system for segmenting the inhomogeneous images by coordinating area force (local and global) term with geodesic edge term in the level set formulation, using the edge scaled method with local and global region-based statistical knowledge to validate the mammogram method images taken from the MIAS miniature dataset. The outcome indicates a precision of $95.85 \%$ [19].

In this study, we proposed a concordance process between a high noise mammogram image (that is, it does not contain constant intensity in different regions) and CVM that requires a constant intensity for each region, as we worked on processing the mammogram images using both the Gaussian filter and CLAHE to obtain a high contrast in the image, we also determined an appropriate value for the parameter $(\mu)$, which is the parameter of the internal energy term in CVM. So that the segmented boundaries can capture tissueinterfering pests easily and accurately. The main goal of the proposed algorithm is to identify tumours of small sizes, for example, calcifications, whose sizes range between $0.05-1 \mathrm{~mm}$, in addition to their location close to the surrounding tissues, so most algorithms find it very difficult to segment it. The accurate identification of this type of tumour, the chances of early detection of breast cancer increase, as there is a large correlation between the presence of calcifications and breast cancer, as detection is necessary for early detection of most breast cancers.

\section{WORK BACKGROUND}

\subsection{The CVM}

The CVM was originally proposed to mitigate the Mumford-Shah problem [20]. CVM is an approach that is capable of segmenting regions without searching for edges using a static, multi-phase model [16], that is based on properties of regions and capable of segmenting regions based on those properties relying on gradients (i.e., edges) to detect region borders. Although the Chan-Vese scheme is based on region properties, it can also detect regions separated by edges. Via several iterative measurements, it can discern within from outside through an arbitrary contour of evolution found in the image to create the required energy function of the model to achieve the minimum. This method approximated each area's image intensity, called $c_{1}$ and $c_{2}$, respectively, within and outside the contour suppose $f(x, y)$ is image, $\Phi(\mathrm{x}, \mathrm{y}) \mathrm{a}$ "level set function" and $C$ an enclosed contour relating to the "zero-level set": $C=\{(x, y) \mid \Phi(x, y)=0\}$. The energy functional $\mathrm{E}_{\mathrm{CVM}}\left(\mathrm{C}, \mathrm{c}_{1}, \mathrm{c}_{2}\right)$ is calculated using the following [19]: 


$$
\begin{aligned}
& \mathrm{E}_{\mathrm{CVM}}\left(\mathrm{C}, \mathrm{c}_{1}, \mathrm{c}_{2}\right)=\lambda_{1} \int_{\Omega}\left|\mathrm{f}(\mathrm{x}, \mathrm{y})-\mathrm{c}_{1}\right|^{2} \mathrm{H}_{\varepsilon}(\Phi(\mathrm{x}, \mathrm{y})) \mathrm{dxdy}+\lambda_{2} \int_{\Omega}\left|\mathrm{f}(\mathrm{x}, \mathrm{y})-\mathrm{c}_{2}\right|^{2}(1- \\
& \left.\mathrm{H}_{\varepsilon}(\Phi(\mathrm{x}, \mathrm{y}))\right) \mathrm{dxdy}+\mu \int_{\Omega} \mid \nabla \mathrm{H}_{\varepsilon}\left(\left.\Phi(\mathrm{x}, \mathrm{y})\right|^{2} \mathrm{dxdy}+v \int_{\Omega} \mathrm{H}_{\varepsilon}(\Phi(\mathrm{x}, \mathrm{y}) \mathrm{dxdy}\right.
\end{aligned}
$$

where $\Omega$ is the image region, $\lambda_{1}, \lambda_{2}, v, \mu$ are positive parameters governing each term's power, where $\lambda$ is fit weight the preferred settings are $\lambda_{1}, \lambda_{2}=1$, and $(\mu)$ is a parameter that controls the length of the contour when decreasing the length of the contour, the contour continues to be smoothed. And constant, $v$ balances the area term for $C$, as it speeds up the evolution of the zero-level set, and is necessary when preparing the perimeter away from the object. $\mathrm{H}_{\varepsilon}(\Phi(\mathrm{x}, \mathrm{y}))$ is the smooth Heaviside function approximation [21]:

$$
\mathrm{H}_{\varepsilon}(\Phi(\mathrm{x}, \mathrm{y}))=\frac{1}{2}\left[1+\frac{2}{\pi} \arctan \left(\frac{\Phi(\mathrm{x}, \mathrm{y})}{\varepsilon}\right)\right]
$$

$\varepsilon$ is regularization constant, Manages the smooth operation of Heaviside. Average global region intensities within and outside of contour $C$ are $c_{1}$ and $c_{2}$, respectively, in (1). We have the appropriate level set formulation by obtaining the derivative of (1) with respect to $\Phi$ using the gradient descent method [22]:

$$
\frac{\partial \Phi(\mathrm{x}, \mathrm{y})}{\partial \mathrm{t}}=\left(-\lambda_{1}\left(\mathrm{f}\left(\mathrm{x}, \mathrm{y}-\mathrm{c}_{1}\right)^{2}+\lambda_{2}\left(\mathrm{f}(\mathrm{x}, \mathrm{y})-\mathrm{c}_{2}\right)^{2}+\mu \operatorname{div}\left(\frac{\nabla \Phi(\mathrm{x}, \mathrm{y})}{|\nabla \Phi(\mathrm{x}, \mathrm{y})|}\right)-v\right) \delta_{\varepsilon}(\Phi(\mathrm{x}, \mathrm{y}))\right.
$$

where $\delta_{\varepsilon}(\Phi(\mathrm{x}, \mathrm{y}))$ is a smooth version of a function of the Dirac delta, explained as [23]:

$$
\delta_{\varepsilon}(\Phi(\mathrm{x}, \mathrm{y}))=\frac{1}{\pi} \frac{\varepsilon}{\varepsilon^{2}+\Phi^{2}}
$$

Besides scaling the smoothness of a Heaviside function in (2), $\varepsilon$ likewise balances the size of the Dirac function in (4). Keeping $\Phi(\mathrm{x}, \mathrm{y})$ constant and minimizing energy in (1) with respect to $c_{1}$ and $c_{2}$, we get:

$$
\begin{aligned}
& \mathrm{c}_{1}=\frac{\int_{\Omega} \mathrm{f}(\mathrm{x}, \mathrm{y}) \mathrm{H}_{\varepsilon}(\Phi(\mathrm{x}, \mathrm{y})) \mathrm{dxdy}}{\int_{\Omega} \mathrm{H}_{\mathcal{\varepsilon}}(\Phi(\mathrm{x}, \mathrm{y})) \mathrm{dxdy}} \\
& \mathrm{c}_{2}=\frac{\int_{\Omega} \mathrm{f}(\mathrm{x}, \mathrm{y})\left(1-\mathrm{H}_{\varepsilon}(\Phi(\mathrm{x}, \mathrm{y}))\right) \mathrm{dxdy}}{\int_{\Omega}\left(1-\mathrm{H}_{\varepsilon}(\Phi(\mathrm{x}, \mathrm{y}))\right) d \mathrm{xdy}}
\end{aligned}
$$

Only global intensity characteristics of an image are correlated with the energy functional in CVM, and this approach can segment only homogeneous regions.

\subsection{Pre-processing}

Low contrast and noise issues are two big issues associated with medical images in the design of a medical image processing algorithm. Research has shown that the mammogram image undergoing active contouring without going through a pre-processing yield a bad outcome and that lesions are not completely recognized [16]. The required pre-processing filter must maintain the normal density of the image when eliminating noise [24]. The suggested method uses a Gaussian filter, which is a filtering approach based on peak detection. The peak measurement is carried out on the basis that peaks are to pulse. The significant factor is that this filter corrects the spectral coefficient of interest and all amplitude spectrum coefficients within the filter window. It is a linear low pass filter, and greater significance is given to the pixels near the edge, thus reducing-edge blurring. The degree of smoothening is controllable, and computing efficiency is also in this filter [25].

\subsection{Image enhancement}

Image enhancement is added to the image after eliminating unnecessary noise in the digital mammography frame. Contrast improvement is a method to enhance the image's consistency for an enhanced overall appearance for post-processing. In this case, contrast enhancement is necessary for image segmentation to improve the mammography image [26]. "Contrast limited adaptive histogram equalization" (CLAHE) is a technique that enhances the poor contrast problem of digital images, particularly medical images [27]. Instead of the whole image, CLAHE works on a specific area, called a tile. The contrast of each tile is improved. The histogram of the output area roughly matches the histogram described by the distribution parameter to remove arbitrarily induced borders. The adjacent tiles are then mixed using bilinear 
interpolation. The contrast can be reduced, especially in homogeneous areas to avoid amplifying any noise that may be existing in the image.

\subsection{Image dataset}

The mammogram included in this study is an acquisition of the "mammographic image analysis society" (MIAS) [28]. It contains primarily 322 images: 209 normal and 113 abnormal images are available. All images size is $1024 \times 1024$ pixels and (.pgm) format. In this dataset, the ground truths of abnormal regions were defined by position and radius. So, in the beginning, we draw circular regions using the embedded information. Region of interest (ROI) can be drawn inside the circular regions manually using a hand-based freehand tool in the software program.

\section{PROPOSED METHODOLOGY}

The proposed technique of tumour segmentation is shown in Figure 1. Due to the nature of the mammogram images we referred to earlier, it is difficult for the default active contour method to handle raw mammogram images.

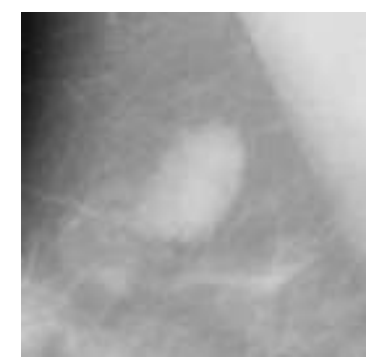

(a)

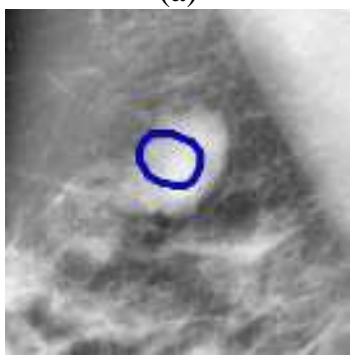

(d)

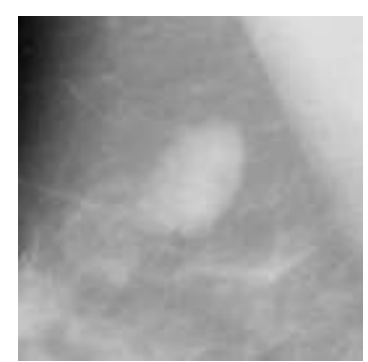

(b)



(e)

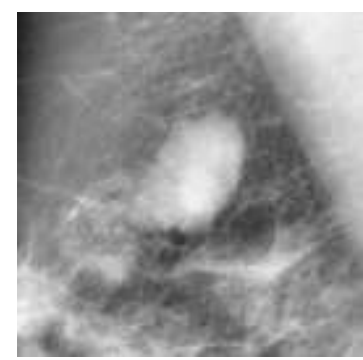

(c)

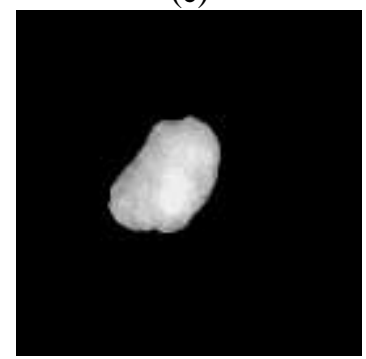

(f)

Figure 1. Block diagram of the proposed model (a) Original image mdb015, (b) After gaussian filter, (c) After gaussian filter \& CLAHE (d) Initial contour (e) Final segmented resulted (f) Segmented tumour

Because it uses external energy that deals only with the contrast of grey levels in the foreground and background to lead the specified zero level towards the boundaries of the object. So, the pre-processing and adjust the definition of the active contour parameters are among the essentials of this project. After trial and comparison, a gaussian filter was chosen for the pre-processing stage and the CLAHE filter for image contrast enhancement to detect abnormal areas. The active contour algorithm contains many input arguments, including the image to be segmented, mask (initial contour position), and several iterations. Firstly, images (abnormal mammogram) are entered to read sequentially by creating a loop in the program. Secondly, the pre-processing step by contrast enhancement filters before going through the active contour equation. Thirdly, take ROI (initial contour) by choosing the freehand tool allows the possibility to define the mask in a flexible manner that fits with different tumour shapes and sizes. Fourthly argument $(N)$ is the maximum number of iterations, it depends on the size of the tumour, as small tumours require fewer iteration than large tumours. Fifthly, the input argument smooth factor $(\mu)$ was defined as the degree of the smoothness of the segmented regions' borders or regularity. After the experiment, $(\mu=3)$ was chosen as the most appropriate value and all used parameters in this work shown in Table 1. 
Table 1. Parameters used in the experiment

\begin{tabular}{clc}
\hline Symbol & Quantity & Parameter value \\
\hline$\lambda_{\mathbf{1}}=\lambda_{\mathbf{2}}$ & fit weight & 1 \\
$\boldsymbol{v}$ & Contraction bias & 0 \\
$\boldsymbol{\mu}$ & smooth factor & 3 \\
$\mathbf{N}$ & maximum number of iterations & 300 \\
$\boldsymbol{\sigma}$ & standard deviation & 0.6 \\
$\boldsymbol{\chi}$ & Row vector & 3 \\
$\mathbf{y}$ & column vector & 3 \\
\hline
\end{tabular}

\section{EXPERIMENT ANALYSIS AND RESULTS}

\subsection{Evaluation metrics}

There are many evaluation criteria for comparing the proposed model with a segmentation technique. These criteria determine the performance of the proposed model by selecting the compatibility between the grand truth $\left(\mathrm{R}_{\mathrm{GT}}\right)$, which represents the region manually segmented by an expert radiologist, and the region resulting from the application of the proposed model $\left(R_{S E G}\right)$. $\left|R_{S E G} \cap R_{G T}\right|$ the number of pixels from the common area of the $\left(R_{G T}\right)$ and $\left(R_{S E G}\right)$ while $\left|R_{S E G} \cup R_{G T}\right|$ the number of all pixels found in the $\left(\mathrm{R}_{\mathrm{GT}}\right)$ and $\left(\mathrm{R}_{\mathrm{SEG}}\right)$. Those criteria take advantage of the following regions and shown in Figure 2:

True Positive $\left(\mathrm{T}_{\mathrm{P}}\right)=\mathrm{R}_{\mathrm{GT}} \cap \mathrm{R}_{\mathrm{SEG}} ;$ False Positive $\left(\mathrm{F}_{\mathrm{P}}\right)=\overline{\mathrm{R}}_{\mathrm{GT}} \cap \mathrm{R}_{\mathrm{SEG}} ;$ False Negative $\left(\mathrm{F}_{\mathrm{N}}\right)=\mathrm{R}_{\mathrm{GT}} \cap \overline{\mathrm{R}}_{\mathrm{SEG}}$

where $T_{P}$ is the number of pixels that belong to the tumour and the proposed algorithm can identify it, $F_{N}$ represents the number of pixels that belong to the tumour and were not recognized by the proposed algorithm, $\mathrm{F}_{\mathrm{p}}$ is the number of pixels that do not belong to the tumour and were diagnosed as a tumour by the proposed algorithm.

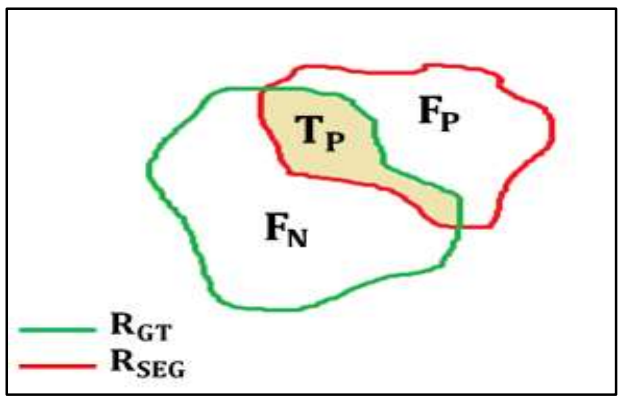

Figure 2. The different regions for the assessment of segmentation

To evaluation the output of the proposed segmentation algorithm, Jaccard coefficients $\left(\mathrm{J}_{\mathrm{S}}\right)$ and Dice coefficients $\left(D_{S}\right)$ are determined. The Jaccard similarity, also known as the area overlap measure, can be defined as:

$$
\mathrm{J}_{\mathrm{S}}=\frac{\left|\mathrm{R}_{\mathrm{SEG}} \cap \mathrm{R}_{\mathrm{GT}}\right|}{\left|\mathrm{R}_{\mathrm{SEG}} \cup \mathrm{R}_{\mathrm{GT}}\right|} \quad \text { This is equivalent to } \quad \mathrm{J}_{\mathrm{S}}=\frac{\mathrm{T}_{\mathrm{P}}}{\mathrm{T}_{\mathrm{P}}+\mathrm{F}_{\mathrm{P}}+\mathrm{F}_{\mathrm{N}}}
$$

Dice coefficients also calculate the degree to which two binary images have spatial overlap.

$$
D_{S}=\frac{2 \times\left|R_{S E G} \cap R_{G T}\right|}{\left|R_{S E G}\right|+\left|R_{G T}\right|} \quad \text { This is equivalent to } \quad D_{S}=\frac{2 \times T_{P}}{2 \times T_{P}+F_{P}+F_{N}}
$$

The $\left(\mathrm{J}_{\mathrm{S}}\right)$ and $\left(\mathrm{D}_{\mathrm{S}}\right)$ values are between 0 (no overlap) and 1 (perfect agreement). The values closer to 1 show an accurate result of segmentation, and the values closer to 0 imply poor segmentation.

PF-Score is the harmonic mean of precision and Sensitivity. Its mathematical expression is as follows:

$$
\mathrm{PF}-\text { Score }=2 \times \frac{\text { precision } \times \text { Sensitivity }}{\text { precision }+ \text { Sensitivity }}
$$


where the precision is the number of correctly identified pixels belonging to the tumour divided by all pixels belonging to the tumour even if not correctly identified.

$$
\text { precision }=\frac{\mathrm{T}_{\mathrm{P}}}{\mathrm{T}_{\mathrm{P}}+\mathrm{F}_{\mathrm{P}}}
$$

and the Sensitivity is the number of correctly identified pixels belonging to the tumour divided by the number of all pixels identified as tumours even if they do not belong to it.

$$
\text { Sensitivity }=\frac{\mathrm{T}_{\mathrm{P}}}{\mathrm{T}_{\mathrm{P}}+\mathrm{F}_{\mathrm{N}}}
$$

The quantitative results give the extent of congruence and difference between $\left(R_{G T}\right)$ and $\left(R_{\mathrm{SEG}}\right)$ in terms of the number of correctly and wrongly diagnosed pixels, but it does not give us an idea of the similarity in terms of shape, so we will use the region's properties to provide us more about the shape of the segmentation results. To describe the congruence and the difference in terms of shape between $\left(\mathrm{R}_{\mathrm{GT}}\right)$ and $\left(R_{\mathrm{SEG}}\right)$, we will use the following area properties (distance, area, diameter, axis). Distance is the distance between the centre $\left(\mathrm{R}_{\mathrm{GT}}\right)$ and $\left(\mathrm{R}_{\mathrm{SEG}}\right)$, the smaller the distance, the higher the accuracy of the measurement. The area is representing the area difference between the two reigns. The smaller is the value, the higher is the congruence; Diameter, which is a value with three possibilities: 1 perfect match, $<1$ this means that $\mathrm{R}_{\mathrm{SEG}}>$ $\mathrm{R}_{\mathrm{GT}},>1$ means that $\mathrm{R}_{\mathrm{SEG}}<\mathrm{R}_{\mathrm{GT}}$. Axis is the difference between the large and small axes of the two areas $\left(\mathrm{R}_{\mathrm{GT}}\right)$ and $\left(\mathrm{R}_{\mathrm{SEG}}\right)$, the congruence is higher when the result approaches zero, i.e., the shape approaches the ball. In all the images included in the study, the colours of the external borders will be blue, green, and red for (ROI, $\mathrm{R}_{\mathrm{GT}}, \mathrm{R}_{\mathrm{SEG}}$ ) respectively, as for the images that represent the region of the segmented tumour only the colours of the regions are white, green, and purple for the regions $\left(\mathrm{T}_{\mathrm{P}}, \mathrm{F}_{\mathrm{P}}, \mathrm{T}_{\mathrm{N}}\right)$ respectively [29].

\subsection{Experimental results and discussion}

The implemented algorithm is by dataset described in subsection (2.4). To evaluate the performance, $\left(\mathrm{R}_{\mathrm{GT}}\right)$ was determined manually by the radiologist. Then the computerized segmentation results were evaluated $\left(\mathrm{R}_{\mathrm{SEG}}\right)$ by a set of evaluation metrics referred to in subsection (4.1). The algorithm showed efficiency in segmenting all abnormal images within the dataset. We worked through pre-processing to solve the problem of inhomogeneous intensity to distinguish the tumour from the background. A large percentage can also improve the performance of CVM if appropriate parameters are specified. The process of organizing the parameters mentioned in Table 1 is an essential matter due to its effect on the final segmentation results.

This study focused on the optimal selection of the parameter $(\mu)$ because of its importance and impact on the results of segmentation. Furthermore, the small values make the contour of the segmentation spread quickly and cannot be controlled, while the high values make the contour of the segmentation not flexible and slow-spreading. So, it needs a very large number of iterations; in both cases, if $(\mu)$ is too big or too small. Therefore, the appropriate determination of this parameter is difficult, especially as we deal with different types of tumours with different characteristics. The best result was returned to the image (mdb315), as shown in Figure 3 and statistically in Table 2. As it is an image of the type circumscribed masses (CIRC), this type of tumour is characterized by clear borders, as the algorithm was able to identify the tumour pixels with very great accuracy according to the indicators (Jaccard, Dice, PF-score, Precision, Sensitivity) that came as follows respectively $(90.1,94.8,95.5,92.1,99.5)$ at iteration (300). As for determining the shape of the tumour and its location, the algorithm was able to determine that very efficiently, as the distance difference between $\left(R_{G T}\right)$ and $\left(R_{S E G}\right)$, is only $(0.16)$ pixels. As for the area, the difference is only $(0.07)$ pixels. As the result of the probability of diameter between them (1.07) that is, $\left(\mathrm{R}_{\mathrm{SEG}}\right)$ less than $\left(\mathrm{R}_{\mathrm{GT}}\right)$ by (0.07) pixels only. As for the congruence between the large and small axis for the two regions it was $(0.14)$ pixels. Meaning that all the results indicate an excellent match between the two regions.

What is new in the proposed algorithm is that it not only can segment large tumours, but it has also been able to efficiently segment breast lesions that are small in size and have low contrast with surrounding breast tissue such as calcifications, where Figure 4 represents an image of a tumour of the type calcifications (CALC). The statistical results in Table 2 indicated superiority great in identifying tumour pixels. For the region characteristics indicators, the results proved that the algorithm was able to determine the location and shape to a degree close to ideal. For example, the deviation of the segmentation result from the complete compatibility between $\left(\mathrm{R}_{\mathrm{GT}}\right)$ and $\left(\mathrm{R}_{\mathrm{SEG}}\right)$, was only $(0.004)$ pixels, meaning they were almost identical. The rest of the results have been filled in Table 2. As we mentioned earlier, large tumours need a large number of iterations to reach a complete determination as (mdb315) need (300) iteration while (mdb265) only need (70) because it is one of the smaller tumours. 


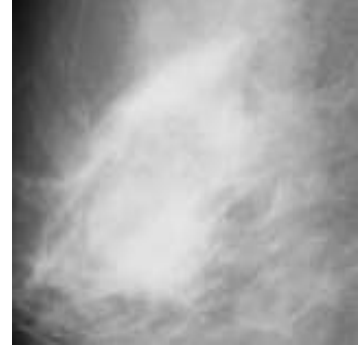

(a)

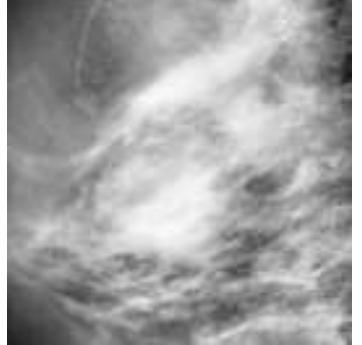

(b)

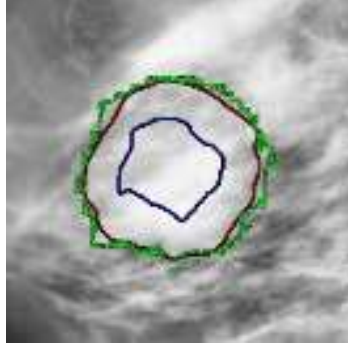

(c)

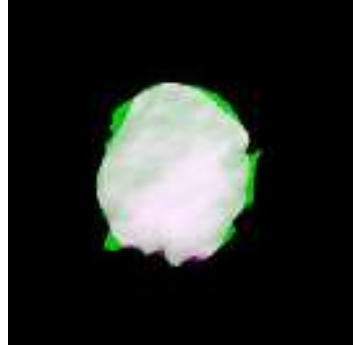

(d)

Figure 3. Segmentation result of the image (mdb315) at iteration 300, (a) original image, (b) after Pre-processing, (c) final result (d) region segmentation of image

Table 2. Measurements (jaccard, dice, pf-score, precision, sensitivity, iteration, and region properties) for images

\begin{tabular}{ccccccccccc}
\hline \multirow{2}{*}{ Image } & Iteration & $\begin{array}{c}\text { Jaccard } \\
\%\end{array}$ & $\begin{array}{c}\text { Similarity } \\
\text { Dice } \\
\%\end{array}$ & $\begin{array}{c}\text { PF- } \\
\text { Score } \%\end{array}$ & $\begin{array}{c}\text { Precision } \\
\%\end{array}$ & $\begin{array}{c}\text { Sensitivity } \\
\%\end{array}$ & Distance & Area & Diameter & Axis \\
\hline mdb315 & 300 & 90.1 & 94.8 & 95.5 & 92.1 & 99.5 & 0.16 & 0.07 & 1.07 & 0.14 \\
mdb256 & 70 & 79.1 & 88.3 & 65.9 & 59.6 & 73.8 & 2.373 & 0.009 & 0.996 & 0.162 \\
\hline
\end{tabular}

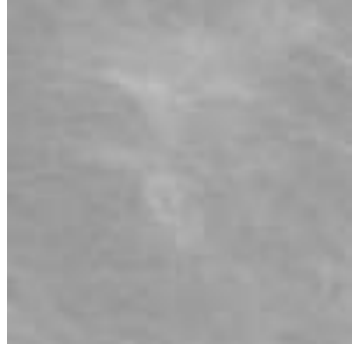

(a)

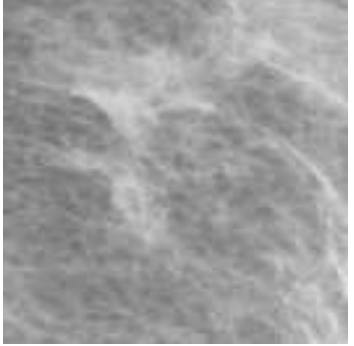

(b)

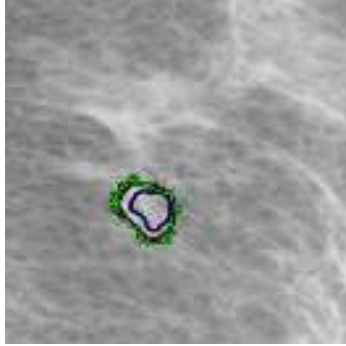

(c)

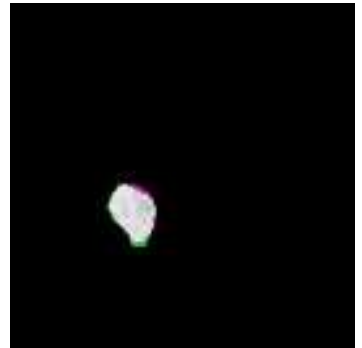

(d)

Figure 4. Segmentation result of the image (mdb265) at iteration 70, (a) original image, (b) after Pre-processing, (c) final result (d) region segmentation of image

\subsection{Comparison with existing techniques}

There is great difficulty in comparing current work with other techniques. The authors use a different number of mammogram samples from different data sets, public and private, and applying measurement criteria that differ from one study to another. The comparison techniques based on active contour models (ACMs) and others without active contour models (WACMs), as shown in Table 3. We compared the proposed algorithm with the work of A. Niaz et al. [30], where they included a p-Laplace term with CVM, they tested it on only 25 mammogram images. A comparison is also made with the algorithm of M. Hmida et al. [18], where the algorithm was a hybrid between CVM and fuzzy c-means, they dealt with 57 mammogram images of the class of masses only. We also provided a comparison with WACMs to prove the reliability of our work. The comparison was made with the work submitted by M. Y. Kamil et al. [31], proposed two algorithms fuzzy C-mean and K-mean. The algorithms were implemented on all database images. Our algorithm showed superiority over all the previously mentioned algorithms according to the statistical results. In addition to our algorithm being unique to successfully deal with all tumours in the database, we took an example of difficult to segmented tumours calcifications. Whereas, the two previous studies ACMs did not deal with all database images if this type of tumour was not segmented. As for the last study mentioned, the segmentation results were weaker with this type of tumour. This is due to the appropriate combination of pre-processing methods and the appropriate selection of CVM parameters. 
Table 3. Statistical results of evaluation on the MIAS dataset

\begin{tabular}{|c|c|c|c|c|c|}
\hline & Method & Ref. & image & Sensitivity\% & Precision\% \\
\hline \multirow{5}{*}{$\sum_{0}^{\infty}$} & $\sum^{\infty}$ & [30] & 25 & - & 98.0 \\
\hline & $\ll$ & [18] & 57 & 91.12 & 88.08 \\
\hline & $\begin{array}{c}\text { Fuzzy C- } \\
\text { mean }\end{array}$ & \multirow{3}{*}{ [31] } & 102 & 99.4 & - \\
\hline & K-mean & & 102 & 98.9 & - \\
\hline & $\begin{array}{c}\text { proposed } \\
\text { method }\end{array}$ & & 102 & 99.5 & 92.1 \\
\hline
\end{tabular}

\section{CONCLUSION}

In this study, we worked to reduce the contrast between breast regions and the prominence of tumour margins by using a gaussian filter and CLAHE as a pre-processing before going through the segmentation stage. Then we worked to adjust the segmentation contour movement CVM by setting an appropriate value for $(\mu=3)$, this value made the contour move freely to trace the boundaries of the tumour with the keep on it from leakage. The results showed that our algorithm scored a superiority in the segmentation of different types of tumours, including tumours of small sizes. The statistical results returned on the highest segmented image are as follows Jaccard=90.1 and Dace=94.8. It is observed that the proposed method was effective and could be used as CAD systems to identify tumours.

\section{REFERENCES}

[1] M. S. Hossain, "Microc alcification Segmentation Using Modified U-net Segmentation Network from Mammogram Images," Journal of King Saud University-Computer and Information Sciences, 2019, doi: 10.1016/j.jksuci.2019.10.014.

[2] R. M. Mann, C. K. Kuhl, K. Kinkel, and C. Boetes, "Breast MRI: guidelines from the European society of breast imaging," European radiology, vol. 18, no. 7, pp. 1307-1318, 2008, doi: 10.1007/s00330-008-0863-7.

[3] B. Chen and R. Ning, "Cone-beam volume CT breast imaging: Feasibility study," Medical physics, vol. 29, no. 5, pp. 755-770, 2002, doi: 10.1118/1.1461843.

[4] O. Olsen and P. C. Gøtzsche, "Cochrane review on screening for breast cancer with mammography," The Lancet, vol. 358, no. 9290, pp. 1340-1342, 2001, doi: 10.1016/S0140-6736(01)06449-2.

[5] Q. Huang, Y. Luo, and Q. Zhang, "Breast ultrasound image segmentation: a survey," International journal of computer assisted radiology surgery, vol. 12, no. 3, pp. 493-507, 2017, doi: 10.1007/s11548-016-1513-1.

[6] A. H. Farhan and M. Y. Kamil, "Texture Analysis of Mammogram Using Local Binary Pattern Method," in Journal of Physics: Conference Series, vol. 1530, no. 1, 2020, p. 012091, doi: 10.1088/1742-6596/1530/1/012091.

[7] B. Pardamean, T. W. Cenggoro, R. Rahutomo, A. Budiarto, and E. K. Karuppiah, "Transfer learning from chest Xray pre-trained convolutional neural network for learning mammogram data," Procedia Computer Science, vol. 135, pp. 400-407, 2018, doi: 10.1016/j.procs.2018.08.190.

[8] Z. Suhail and R. Zwiggelaar, "Histogram-based approach for mass segmentation in mammograms," in 15th International Workshop on Breast Imaging (IWBI2020), International Society for Optics and Photonics, vol. 11513, 2020, p. 1151325 , doi: $10.1117 / 12.2563621$.

[9] R. Jin and G. Weng, "Active contour model based on improved fuzzy c-means algorithm and adaptive functions," Computers Mathematics with Applications, vol. 78, no. 11, pp. 3678-3691, 2019, doi: 10.1016/j.camwa.2019.06.010.

[10] T. Heimann and H. Delingette, "Model-based segmentation," in Biomedical Image Processing: Springer, 2010, pp. 279-303, doi: 10.1007/978-3-642-15816-2_11.

[11] T. McInerney and D. Terzopoulos, "Deformable models in medical image analysis: a survey," Medical image analysis, vol. 1, no. 2, pp. 91-108, 1996.

[12] G. Huo, S. X. Yang, Q. Li, and Y. Zhou, "A Robust and Fast Method for Sidescan Sonar Image Segmentation Using Nonlocal Despeckling and Active Contour Model," IEEE Transactions on Cybernetics, vol. 47, no. 4, pp. 855-872, 2017, doi: 10.1109/TCYB.2016.2530786.

[13] X. Yang, X. Gao, D. Tao, X. Li, and J. Li, "An efficient MRF embedded level set method for image segmentation," IEEE transactions on image processing, vol. 24, no. 1, pp. 9-21, 2014, doi: 10.1109/TIP.2014.2372615.

[14] C. Xiu, H. Yin, and Y. Liu, "Image Segmentation of CV Model Combined with Sobel Operator," in 2020 Chinese Control And Decision Conference (CCDC), 22-24 Aug. 2020 2020, pp. 4356-4360, doi: 10.1109/CCDC49329.2020.9164450.

[15] S. N. Acho and W. I. D. Rae, "Interactive breast mass segmentation using a convex active contour model with optimal threshold values," Physica Medica, vol. 32, no. 10, pp. 1352-1359, 2016, doi: 10.1016/j.ejmp.2016.05.054.

[16] M. Mustafa, H. N. O. Rashid, N. R. H. Abdullah, R. Samad, and D. Pebrianti, "Mammography image segmentation: Chan-Vese active contour and localised active contour approach," Indonesian Journal of Electrical Engineering Computer Science, vol. 5, no. 3, pp. 577-588, 2017, doi: 10.11591/ijeecs.v5.i3.pp577-583. 
[17] S. Soomro and K. N. Choi, "Robust active contours for mammogram image segmentation," in 2017 IEEE International Conference on Image Processing (ICIP), 2017, pp. 2149-2153, doi: 10.1109/ICIP.2017.8296662.

[18] M. Hmida, K. Hamrouni, B. Solaiman, and S. Boussetta, "Mammographic mass segmentation using fuzzy contours," Computer methods programs in biomedicine, vol. 164, pp. 131-142, 2018, doi: 10.1016/j.cmpb.2018.07.005.

[19] S. Soomro, A. Munir, and K. N. Choi, "Fuzzy c-means clustering based active contour model driven by edge scaled region information," Expert Systems with Applications, vol. 120, pp. 387-396, 2019, doi: 10.1016/j.eswa.2018.10.052.

[20] T. F. Chan and L. A. Vese, "Active contours without edges," IEEE Transactions on image processing, vol. 10, no. 2, pp. 266-277, 2001, doi: 10.1109/83.902291.

[21] J. Liao et al., "The Design and Implementation of Plant Disease Spot Segmentation Algorithm Based on Improved CV Model," in 2019 2nd International Conference on Safety Produce Informatization (IICSPI), IEEE, 2019, pp. 602-605, doi: 10.1109/IICSPI48186.2019.9095875.

[22] M. M. Abdelsamea, G. Gnecco, and M. M. Gaber, "An efficient Self-Organizing Active Contour model for image segmentation," Neurocomputing, vol. 149, pp. 820-835, 2015, doi: 10.1016/j.neucom.2014.07.052.

[23] Z. Wang, K. Wang, F. Yang, S. Pan, and Y. Han, "Image segmentation of overlapping leaves based on Chan-Vese model and Sobel operator," Information processing in agriculture, vol. 5, no. 1, pp. 1-10, 2018, doi: 10.1016/j.inpa.2017.09.005.

[24] S. J. S. Gardezi, A. Elazab, B. Lei, and T. Wang, "Breast cancer detection and diagnosis using mammographic data: systematic review," Journal of medical Internet research, vol. 21, no. 7, 2019, doi: 10.2196/14464.

[25] A. M. Joseph, M. G. John, and A. S. Dhas, "Mammogram image denoising filters: A comparative study," in 2017 Conference on Emerging Devices and Smart Systems (ICEDSS), IEEE, 2017, pp. 184-189, doi: 10.1109/ICEDSS.2017.8073679.

[26] M. Y. Kamil, "Computer-aided diagnosis system for breast cancer based on the Gabor filter technique," International Journal of Electrical and Computer Engineering (IJECE), vol. 10, no. 5, pp. 5235-5242, 2020, doi: 10.11591/ijece.v10i5.pp5235-5242.

[27] S. Sahu, A. K. Singh, S. Ghrera, and M. y. Elhoseny, "An approach for de-noising and contrast enhancement of retinal fundus image using CLAHE," Optics Laser Technology, vol. 110, pp. 87-98, 2019, doi: 10.1016/j.optlastec.2018.06.061.

[28] J. Suckling, "site "The mini-MIAS database of mammograms" [http://peipa. essex. ac. uk/info/mias. html]," Último acesso: Agosto, 2010.

[29] M. Y. Kamil, "A deep learning framework to detect Covid-19 disease via chest X-ray and CT scan images," International Journal of Electrical \& Computer Engineering, vol. 11, no. 1, pp. 2088-8708, 2021, doi: 10.11591/ijece.v11i1.pp844-850.

[30] A. Niaz et al., "Inhomogeneous Image Segmentation Using Hybrid Active Contours Model With Application to Breast Tumor Detection," IEEE Access, vol. 8, pp. 186851-186861, 2020, doi: 10.1109/ACCESS.2020.3029333.

[31] M. Y. Kamil and A. M. Salih, "Mammography Images Segmentation via Fuzzy C-mean and K-mean," International Journal of Intelligent Engineering Systems, vol. 12, no. 1, pp. 22-29, 2019, doi: 10.22266/ijies2019.0228.03.

\section{BIOGRAPHIES OF AUTHORS}
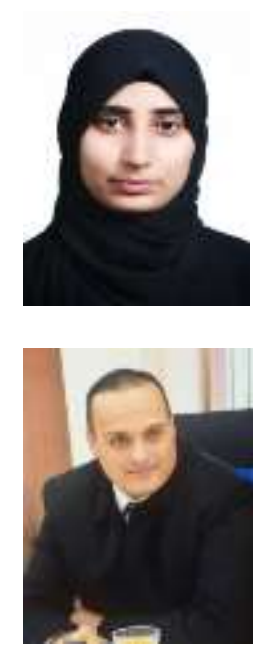

Eman A. Radhi received the B.S. degree in physics science from Wasit University, Iraq. She is currently pursuing the M.S. degree with the digital image processing, Mustansiriya University, Iraq. Her research interests include image segmentation, image recognition, and medical imaging.

Mohammed Y. Kamil obtained his M. Sc. in Optics from Mustansiriya University, College of Science, Physics department, Iraq, in 2005. He received his Ph.D. in digital image processing from Mustansiriya University in 2011. He is interested in medical image processing, computer vision, and Artificial Intelligence. He is a member of the IEEE Iraq section. 